\title{
Marangoni Boundary Layer Flow and Heat Transfer of Graphene-Water Nanofluid with Particle Shape Effects
}

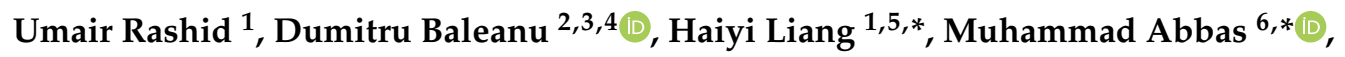 \\ Azhar Iqbal ${ }^{7}$ (D) and Jamshid ul Rahman ${ }^{8}$ \\ 1 CAS Key Laboratory of Mechanical Behavior and Design of Materials, Department of Modern Mechanics, \\ University of Science and Technology of China, Hefei 230026, Anhui, China; umair2014@mail.ustc.edu.cn \\ 2 Department of Mathematics, Faculty of Arts and Sciences, Cankaya University, Ankara 06530, Turkey; \\ dumitru@cankaya.edu.tr \\ 3 Department of Medical Research, China Medical University, Taichung 40402, Taiwan \\ 4 Institute of Space Sciences, 077125 Magurele, Romania \\ 5 IAT-Chungu Joint Laboratory for Additive Manufacturing, Anhui Chungu 3D Printing Institute of \\ Intelligent Equipment and Industrial Technology, Wuhu 241200, Anhui, China \\ 6 Department of Mathematics, University of Sargodha, Sargodha 40100, Pakistan \\ 7 Mathematics and Natural Sciences, Prince Mohammad Bin Fahd University, Al Khobar 31952, Saudi Arabia; \\ aiqbal@pmu.edu.sa \\ 8 Basic Science Department, Balochistan University of Engineering and Technology Khuzdar, Khuzdar 89100, \\ Pakistan; jamshaid.rahman@buetk.edu.pk \\ * Correspondence: hyliang@ustc.edu.cn (H.L.); muhammad.abbas@uos.edu.pk (M.A.)
}

Received: 21 July 2020; Accepted: 26 August 2020; Published: 9 September 2020

check for updates

\begin{abstract}
Graphene nanofluids have attracted the attention of many researchers because of a variety of remarkable properties such as extraordinary electronic transport properties, high thermal conductivity, and large specific surface areas. This paper investigates the shape effects of nanoparticles on the Marangoni boundary layer of graphene-water nanofluid flow and heat transfer over a porous medium under the influences of the suction parameter. The graphene-water nanofluid flow was contained with various shapes of nanoparticles, namely sphere, column, platelet, and lamina. The problem is modeled in form of partial differential equations (PDES) with boundary conditions. The governing transport equations are converted into dimensionless form with the help of some suitable nondimensional variables. The solution of the problem was found numerically using the NDSolve technique of Mathematica 10.3 software. In addition, the numerical solutions were also compared with analytical results. The homotopy analysis method (HAM) is used to calculate the analytical results. The results show that lamina-shaped nanoparticles have better performance on temperature distribution while sphere-shaped nanoparticles are more efficient for heat transfer than other shapes of nanoparticles.
\end{abstract}

Keywords: numerical solution; porous medium; nanoparticles; nanofluids; Marangoni boundary layer flow

\section{Introduction}

Due to the abovementioned outstanding thermophysical properties of graphene, it has become a solid candidate in the field of fluid heat transfer. The work of Novoselov et al. [1] in 2004 is pioneering in its discussion of graphene. Graphene-based nanofluids have high heat transfer and thermal conductivity characteristics as compared to other carbon materials [2]. Graphene is mostly used as a nanofluid. A water-graphene nanofluid is preferable because it is a good coolant for real-time 
applications of heat transfer [3]. Many researchers have worked on graphene nanofluid flow due to its outstanding thermophysical properties. Alay [4] examined the exact solution of water-graphene nanofluid over a shrinking/stretching sheet. Upadhya et al. [5] numerically discussed water-graphene nanofluid flow over a stretching cylinder and also performed a comparison with silver-water nanofluid. Upadhya et al. [6] studied theoretically the unsteady flow of Carreau fluid in a suspension of graphene and dust nanoparticles with Cattaneo-Christov heat flux. Noor et al. [7] discussed an analytical solution of graphene nanofluid and heat transfer in a thin film. Shit and Mukherjee [8] examined graphene-polydimethylsiloxane nanofluid between two plates with the thermal radiation effect. Arif et al. [9] studied the analytical results of Maxwell fluid flow by using molybdenum disulfide and graphene nanoparticles in engine oil. Mankiandan and Baskar [10] discussed the thermal conductivity of water-graphene nanofluid using factorial design experiments.

Very challenging scientific phenomena and problems are displayed by nonlinear ordinary differential equations (ODEs) or partial differential equations (PDEs). For instance, boundary layer fluid flow can be mentioned as an example. The research on several techniques applied for solving nonlinear differential equations (DEs) is a significant topic for analyzing mathematical problems related to engineering. There are various techniques for solving nonlinear equations that range from completely numerical to completely analytical ones. Besides the many advantages of applying numerical techniques, closed results seem more appealing because they explain physical insights through the physical problem. By using an analytical method, parametric studies become more convenient [11]. Some of these techniques include the variation iteration method [12], Galerkin method [13], optimal homotopy asymptotic method [14], homotopy analysis method (HAM) [15], differential transformation method [16], homotopy perturbation method [17], and Adomian decomposition method [18].

Marangoni convection flow appears with the change of surface tension along liquid-gas or liquid-liquid interfaces, which was first observed by Napolitan $[19,20]$. Moreover, it is also significant in daily life uses such as biomedical and industrial fields, coating flow technology, film drainage in emulsions, the drying of semi-conductor wafers in microelectronics, foams, microfluidics, and surfactant replacement therapy for neonatal infants [19]. Most significantly, Marangoni convection has been demonstrated using the boiling test in microgravity; likewise, it is also significant in the absence of gravity [21,22]. Pop et al. [23] examined Marangoni convection phenomena. Many researchers have discussed Marangoni convection by considering different geometry shapes [23-27].

Arafune and Hirata [28] discussed an analytical solution for the velocity profile of Marangoni convection by considering constant velocity at any point on the free surface. Various researchers studied the different physical effects of Marangoni convection boundary layer flow by taking the thermophysical properties model without any effect of the Prandtl number on base fluid and nanoparticles [29-32]. Marangoni convection is a significant part of the determination of fluid movement and material processing, especially low gravity hydrodynamics, to analyze mass and heat transfer interaction [33]. Many researchers have worked on heat transfer in Marangoni convection flow. Marangoni convection showed a dominant role played in heat transfer and flow properties of fluid due to the change in liquid surface tension in a temperature gradient field regardless of system size. Marangoni convection and heat transfer in non-Newtonian power-law fluid while imposing linear surface temperature have been explored [34]. The Prandtl number effect was studied by Christopher and Wang [25], and they ascertained the analytical solution of the temperature profile by considering Marangoni convection over a flat surface. Lin and Zheng [35] studied the heat transfer in Cu-water Marangoni boundary layer nanofluid flow over a disk.

Inspired by the above uses of graphene nanofluids, a graphene-water nanofluid was considered in this study. As far as we are aware, studies on the shape effects of graphene nanoparticles have never been done. The shape of nanoparticles is very significant in the enhancement of heat transfer. It is necessary to find the heat transfer under exact nanoparticle shapes [36]. The main objective of this study is to find the shape effects of graphene nanoparticles in Marangoni boundary layer flow and heat transfer. The effect of the suction parameter under the influences of thermal radiation and a 
magnetic field was also considered. Finally, graphs of velocity, temperature, and the Nusselt number were plotted and all their aspects are debated.

\section{Description of the Problem}

We consider a laminar, incompressible, steady, and two-dimensional Marangoni boundary layer flow of graphene-water nanofluid over a porous surface with the effects of the suction parameter. It is also considered that nanoparticles and base fluid water are in equilibrium and, also, that no slip occurs between them. It is assumed that flow takes place at $y \geq 0$. Furthermore, it is also assumed that surface tension is linear with temperature. A uniform B magnetic field is applied in the normal direction to the surface. The partial governing equations of the problem are modeled as [30]. The thermophysical properties of graphene-water nanofluid are presented in Table 1. The values of empirical shape factor and sphericity for different shapes of nanoparticles are presented in Table 2.

$$
\begin{gathered}
\frac{\partial \mathrm{u}}{\partial \mathrm{x}}+\frac{\partial \mathrm{v}}{\partial \mathrm{y}}=0 \\
\mathrm{u} \frac{\partial \mathrm{u}}{\partial \mathrm{x}}+\mathrm{v} \frac{\partial \mathrm{u}}{\partial \mathrm{y}}=\frac{\mu_{\mathrm{n}_{\mathrm{f}}}}{\rho_{\mathrm{n}_{\mathrm{f}}}} \frac{\partial^{2} \mathrm{u}}{\partial \mathrm{y}^{2}}-\frac{\sigma_{\mathrm{n}_{\mathrm{f}}} \mathrm{B}^{2} u^{2}}{\rho_{n_{f}}}-\frac{\mu_{\mathrm{n}_{\mathrm{f}}}}{\sigma_{\mathrm{n}_{\mathrm{f}}} K} \mathrm{u} \\
u \frac{\partial T}{\partial x}+v \frac{\partial T}{\partial y}=\frac{k_{n_{f}}}{(\rho C p)_{n f}} \frac{\partial^{2} T}{\partial y^{2}}-\frac{1}{(\rho C p)_{n f}} \frac{\partial \mathrm{q}_{\mathrm{r}}}{\partial \mathrm{y}}
\end{gathered}
$$

where $\mathrm{u}$ and $\mathrm{v}$ are components of velocity, along the $x$ axis and $y$ axis, respectively, and $T, \mu_{\mathrm{n}_{\mathrm{f}}} \rho_{\mathrm{n}_{\mathrm{f}}},(\rho C p)_{n f}, \alpha_{\mathrm{n}_{\mathrm{f}}}$ are temperature, effective viscosity, effective density, heat capacity, and thermal diffusivity of nanofluid, respectively. The quantities like viscosity, effective density, heat capacitance, and electrically conductivity of nanofluid are the following as in [4].

$$
\left\{\begin{array}{c}
\rho_{n_{f}}=\rho_{f}(1-\phi)+\rho_{s} \phi, \mu_{n_{f}}=\frac{\mu_{f}}{(1-\phi)^{2 \cdot 5}},(\rho C p)_{n f}=(\rho C p)_{f}(1-\phi)+(\rho C p)_{s} \phi \\
\sigma_{n_{\mathrm{f}}}=1+\frac{3 \phi\left(\frac{\sigma_{s}}{\sigma_{f}}-1\right)}{\left(\frac{\sigma_{s}}{\sigma_{f}}+2\right)-\phi\left(\frac{\sigma_{s}}{\sigma_{f}}-1\right)}
\end{array}\right.
$$

where $f, n f$, and $s$ are denote the thermophysical properties of the base fluid, nanofluid, and nanoparticle solid, respectively. Furthermore, the Hamilton-Crosser model is used for mixtures of thermal conductivity and is effective for both nonspherical and spherical shapes of dispersed nanoparticles. According to Hamilton-Crosser model, the mixture of thermal conductivity can be calculated from the following formula

$$
\mathrm{k}_{\mathrm{n}_{\mathrm{f}}}=\mathrm{k}_{\mathrm{f}}\left[\frac{\mathrm{k}_{\mathrm{s}}+\mathrm{k}_{\mathrm{f}}(m-1)-\left(\mathrm{k}_{\mathrm{f}}-\mathrm{k}_{\mathrm{s}}\right)(m-1) \phi}{\mathrm{k}_{\mathrm{s}}+\mathrm{k}_{\mathrm{f}}(m-1)+\left(\mathrm{k}_{\mathrm{f}}-\mathrm{k}_{\mathrm{s}}\right) \phi}\right]
$$

where $m, \phi$ are the shape factor and sphericity of solid nanoparticle.

Table 1. Thermophysical properties of the water and graphene nanoparticles [4].

\begin{tabular}{ccc}
\hline Physical Properties & Graphene & Pure Water \\
\hline $\mathrm{C}_{\mathrm{p}}(\mathrm{J} / \mathrm{kg} \mathrm{K})$ & 2100 & 4179 \\
$\rho\left(\mathrm{kg} / \mathrm{m}^{3}\right)$ & 2250 & 997.1 \\
$\mathrm{k}\left(\mathrm{W} / \mathrm{m} \mathrm{K}^{3}\right.$ & 2500 & 0.613 \\
$\sigma(\Omega \mathrm{m})^{-1}$ & $1 \times 10^{7}$ & 0.05 \\
\hline
\end{tabular}


Table 2. The values of shape parameters as [37,38].

\begin{tabular}{ccccc}
\hline Shape parameter & Column & Sphere & Platelet & Lamina \\
\hline$\phi$ & 0.4710 & 1 & 0.526 & 0.1857 \\
$\mathrm{~m}$ & 6.3698 & 3 & 5.7 & 16.1576 \\
\hline
\end{tabular}

We have used the Rosseland approximation for radiation. The radiative heat flux in simplified form is expressed as

$$
\mathrm{q}_{\mathrm{r}}=-\frac{\sigma^{*}}{3 k^{*}} \cdot \frac{\partial \mathrm{T}^{4}}{\partial \mathrm{y}}
$$

where $\sigma^{*}$ and $k^{*}$ are the Stefan Boltzmann constant and coefficient of mean absorption, respectively. Further expanding $\mathrm{T}^{4}$ in a Taylor series about $\mathrm{T}_{\infty}$ and neglecting terms of higher order, we get

$$
\mathrm{T}^{4} \approx 4 \mathrm{~T}_{\infty}^{3} \mathrm{~T}-3 \mathrm{~T}_{\infty}^{4}
$$

Equation (3) reduces to

$$
u \frac{\partial T}{\partial x}+v \frac{\partial T}{\partial y}=\frac{k_{n_{f}}}{(\rho C p)_{n f}} \frac{\partial^{2} T}{\partial y^{2}}+\frac{16 \sigma^{*}}{3(\rho C p)_{n f} K^{*}} \frac{\partial^{2} T}{\partial y^{2}} \frac{1}{(\rho C p)_{n f}} \frac{\partial \mathrm{q}_{\mathrm{r}}}{\partial \mathrm{y}} .
$$

The boundary conditions subjected to the problems are

$$
\begin{gathered}
\mu_{\mathrm{n}_{\mathrm{f}}} \frac{\partial \mathrm{u}}{\partial \mathrm{y}}=\frac{\partial \sigma}{\partial \mathrm{T}} \frac{\partial \mathrm{T}}{\partial \sigma}, \mathrm{v}=0, \mathrm{~T}=a x^{2}+\mathrm{T}_{\infty} \text { at } y=0 \\
\mathrm{u}=0, \mathrm{~T}=\mathrm{T}_{\infty} \text { at } \mathrm{y} \rightarrow \infty
\end{gathered}
$$

where $a=\frac{\Delta T}{L} . \Delta T$ and $L$ are constant characteristic temperature and length of surface, respectively.

The stream function $\psi$, similarity variable $\eta$, dimensionless temperature $\theta(\eta)$ and dimensionless velocity $f(\eta)$ are defined as:

$$
\left\{\begin{array}{c}
\psi(\eta)=\xi_{2} x f(\eta) \\
\theta(\eta)=\frac{T-T_{\infty}}{a x^{2}} \\
\eta=\xi_{1} y
\end{array}\right.
$$

where

$$
\xi_{1}=\left(\frac{\sigma_{0 \gamma \alpha \rho_{f}}}{\mu_{f}{ }^{2}}\right)^{\frac{1}{3}}, \xi_{2}=\left(\frac{\sigma_{0 \gamma \alpha \mu_{f}}}{\rho_{f}{ }^{2}}\right)^{\frac{1}{3}}, \mathbf{u}=\frac{\partial \psi}{\partial \mathrm{y}}, v=-\frac{\partial \psi}{\partial \mathbf{x}}
$$

where $T$ and $T_{\infty}$ are the reference temperature and nanofluid temperature far away the wall and $\gamma$ is the surface tension defined as

$$
\gamma=\left[1-\left(\mathrm{T}-\mathrm{T}_{\infty}\right) \bar{\gamma}\right] \gamma_{0}
$$

where $\gamma_{0}$ and $\bar{\gamma}$ are surface tension and surface tension change with temperature, respectively.

Substituting Equations (4), (5), and (10) into Equations (1), (2), and (8), the following nonlinear ODES are obtained

$$
\begin{gathered}
A_{1} f^{\prime \prime \prime}+A_{2}\left(f^{\prime 2}-f^{\prime} f^{\prime \prime}\right)-f^{\prime}\left(A_{3} M+\frac{A_{1}}{\mathrm{Kp}}\right)=0 \\
\frac{1}{A_{5}}\left(\frac{1}{P r} A_{4}+R d\right) \theta^{\prime \prime}+f \theta^{\prime}-2 \theta f^{\prime}=0 .
\end{gathered}
$$

The boundary value equation becomes

$$
f(0)=A, f^{\prime \prime}(0)=-2(1-\phi)^{2 \cdot 5}, f^{\prime}(\infty)=0, \theta(0)=1, \theta(\infty)=0
$$


where A is constant mass transfer parameter, $A>0$ is for suction parameter $r, M=\frac{H_{0 \sigma_{f}}^{2}}{\rho_{f}} \frac{1}{\xi_{1} \xi_{2}}$ denotes the magnetic field, $\mathrm{Kp}=\frac{\mu_{f}}{\rho_{f} k} \frac{1}{\xi_{1} \xi_{2}}$ denotes the permeability parameter, $R d=\frac{16 \sigma^{*} \mathrm{~T}_{\infty}^{3}}{3 \mathrm{k}_{\mathrm{n}_{\mathrm{f}}} k^{*}}$ denotes the radiation parameters, and $\operatorname{Pr}=\frac{v_{\mathrm{f}}}{\alpha_{\mathrm{f}}}$ is the prandtl number.

$$
\left\{\begin{array}{c}
A_{1}=\frac{1}{(1-\phi)^{2 \cdot 5}}, A_{2}=\frac{\rho_{s}}{\rho_{f}} \phi+(1-\phi), A_{3}=\frac{3 \phi\left(\frac{\sigma_{s}}{\sigma_{f}}-1\right)}{\left(2+\frac{\sigma_{s}}{\sigma_{f}}\right)-\left(\frac{\sigma_{s}}{\sigma_{f}}-1\right) \phi}+1, \\
A_{4}=\frac{\left[k_{s}+(m-1) k_{f}\right]-(m-1) \phi\left(k_{f}-k_{s}\right)}{\left[k_{s}+(m-1) k_{f}\right]+\phi\left(k_{f}-k_{s}\right)}, A_{5}=(1-\phi)+\phi \frac{(\rho C p)_{s}}{(\rho C p)_{f}} .
\end{array}\right.
$$

The quantity particle of interest Nusselt number $\mathrm{Nu}$ is given as

$$
\mathrm{Nu}=\frac{x q_{w}(x)}{k_{f}\left[T-T_{\infty}\right]}
$$

where $q_{\mathrm{w}}(x)$ is the wall heat flux given by

$$
q_{w}(x)=-k_{n_{f}}\left(\frac{\partial T}{\partial z}\right)_{y=o}
$$

Using Equation (6) in Equation (13), we get

$$
\mathrm{Nu}=-\left(A_{4}+\mathrm{Rd} * \operatorname{Pr}\right) \xi_{1} x \theta^{\prime}(0)
$$

\section{Solution Methodology}

The initial guesses are defined as

$$
\mathcal{L}_{\mathrm{f}}=\mathrm{f}^{\prime \prime \prime}-\mathrm{f}^{\prime}, \mathcal{L}_{\theta}=\theta^{\prime \prime}-\theta
$$

which satisfied the following properties

$$
\mathcal{L}_{\mathrm{f}}\left[\mathrm{Z}_{1}+\mathrm{Z}_{2} \mathrm{e}^{\eta}+\mathrm{Z}_{3} \mathrm{e}^{-\eta}\right]=0, \mathcal{L}_{\theta}\left[\mathrm{Z}_{4} \mathrm{e}^{\eta}+\mathrm{Z}_{5} \mathrm{e}^{-\eta}\right]=0
$$

where $\mathrm{Z}_{1}, \mathrm{Z}_{2}, \mathrm{Z}_{3}, \mathrm{Z}_{4}$, and $\mathrm{Z}_{5}$ are arbitrary constants.

Let $\mathrm{q} \in[0,1]$ represent an embedding parameter and $\hbar_{1} \neq 0$ and $\hbar_{2} \neq 0$ are the auxiliary linear operators. The generalized homotopic equations corresponding to Equations (12) and (13)

$$
\begin{gathered}
\left\{\begin{array}{c}
(1-q) \mathcal{L}_{\mathrm{f}}\left[\hat{\mathrm{f}}(\eta, q)-\mathrm{f}_{0}(\eta)\right]=q \hbar_{1} \mathcal{N}_{\mathrm{f}}\left[\hat{\mathrm{f}}(\eta, \mathrm{q}), \hat{\theta}_{0}(\eta, \mathrm{q})\right] \\
\hat{\mathrm{f}}(0, \mathrm{q})=f_{w}, \hat{\mathrm{f}}^{\prime \prime}(0, \mathrm{q})=-2(1-\phi)^{2 \cdot 5}, \hat{\mathrm{f}}(\infty, \mathrm{q})=0
\end{array}\right. \\
\left\{\begin{array}{c}
(1-\mathrm{q}) \mathcal{L}_{\theta}\left[\hat{\theta}(\eta, \mathrm{q})-\theta_{0}(\eta)\right]=q \hbar_{2} \mathcal{N}_{\theta}\left[\hat{\theta}_{0}(\eta, \mathrm{q}), \hat{\mathrm{f}}(\eta, \mathrm{q})\right] \\
\hat{\theta}(0, \mathrm{q})=1, \hat{\theta}(\infty, \mathrm{q})=0
\end{array}\right.
\end{gathered}
$$

and

$$
\begin{aligned}
\mathrm{N}_{\mathrm{f}}[\hat{\mathrm{f}}(\eta, \mathrm{q}), \hat{\theta}(\eta, \mathrm{q})] & \\
= & A_{1} \frac{\partial^{3} \hat{\mathrm{f}}(\eta, \mathrm{q})}{\partial \eta^{3}}-A_{2} \hat{\mathrm{f}}(\eta, \mathrm{q}) \frac{\partial^{2} \hat{\mathrm{f}}(\eta, \mathrm{q})}{\partial \eta^{2}}+A_{2} \frac{\partial^{2} \hat{\mathrm{f}}(\eta, \mathrm{q})}{\partial \eta^{2}} \frac{\partial^{2} \hat{\mathrm{f}}(\eta, \mathrm{q})}{\partial \eta^{2}}-\left(A_{3} M\right. \\
+ & \left.\frac{A_{1}}{\mathrm{Kp}}\right) \frac{\partial \hat{\mathrm{f}}(\eta, \mathrm{q})}{\partial \eta}=0 \\
\mathrm{~N}_{\theta}[\hat{\theta}(\eta, \mathrm{q}), \hat{\mathrm{f}}(\eta, \mathrm{q})]= & \frac{1}{A_{5}}\left[\frac{1}{P r} A_{4}+R d\right] \frac{\partial^{2} \hat{\theta}(\eta, \mathrm{q})}{\partial \eta^{2}}+\hat{\mathrm{f}}(\eta, \mathrm{q}) \frac{\partial \hat{\theta}(\eta, \mathrm{q})}{\partial \eta}-2 \frac{\partial \hat{\mathrm{f}}(\eta, \mathrm{q})}{\partial \eta} \hat{\theta}(\eta, \mathrm{q})=0 .
\end{aligned}
$$


Due to Taylor's series, with respect to q, we have

$$
f(\eta, q)=f_{0}(\eta)+\sum_{m=1}^{\infty} f_{m}(\eta) q^{m}, \theta(\eta, q)=\theta_{0}(\eta)+\sum_{m=1}^{\infty} \theta_{m}(\eta) q^{m}
$$

where

$$
f_{m}(\eta)=\left.\frac{1}{m !} \frac{\partial^{m} f(\eta, q)}{\partial \eta^{m}}\right|_{q=0}, \theta m(\eta)=\left.\frac{1}{m !} \frac{\partial^{m} \theta(\eta, q)}{\partial \eta^{m}}\right|_{q=0}
$$

And, thus, higher-order deformation problems are

$$
\begin{gathered}
\left\{\begin{array}{c}
\mathcal{L}_{\mathrm{f}}\left[\mathrm{f}_{\mathrm{m}}(\eta)-\chi_{\mathrm{m}} \mathrm{f}_{\mathrm{m}-1}(\eta)\right]=\hbar_{\mathrm{f}} \mathcal{R}_{\mathrm{f}}^{\mathrm{m}}\left(\mathrm{f}_{\mathrm{m}-1}(\eta), \theta_{\mathrm{m}-1}(\eta)\right) \\
\mathrm{f}_{\mathrm{m}}(0)=0, \mathrm{f}_{\mathrm{m}}^{\prime \prime}(0)=0, \mathrm{f}_{\mathrm{m}}^{\prime}(\infty)=0
\end{array}\right. \\
\left\{\begin{array}{c}
\mathcal{L} \mathrm{L}_{\mathrm{f}}\left[\theta_{\mathrm{m}}(\eta)-\chi_{\mathrm{m}} \theta_{\mathrm{m}-1}(\eta)\right]=\hbar_{\theta} \mathcal{R}_{\theta}^{\mathrm{m}}\left(\theta_{\mathrm{m}-1}(\eta), \mathrm{f}_{\mathrm{m}-1}(\eta)\right) \\
\theta_{\mathrm{m}}(0)=0, \theta_{\mathrm{m}}(\infty)=0 .
\end{array}\right.
\end{gathered}
$$

Here,

$$
\chi_{\mathrm{m}}=\left\{\begin{array}{c}
0 \text { when } \mathrm{m} \leq 1 \\
1, \quad \mathrm{~m}>1
\end{array}\right.
$$

where

$$
\begin{gathered}
\mathcal{R}_{\mathrm{f}}^{\mathrm{m}} \mathrm{f}_{\mathrm{m}}(\eta)=A_{1} \mathrm{f}_{\mathrm{m}-1}^{\prime \prime \prime}(\eta)-A_{2} \sum_{\mathrm{z}=0}^{\mathrm{m}-1} \mathrm{f}_{\mathrm{z}}^{\prime} \mathrm{f}_{\mathrm{m}-1-\mathrm{z}}^{\prime \prime}+A_{2} \sum_{\mathrm{z}=0}^{\mathrm{m}-1} \mathrm{f}_{\mathrm{z}}^{\prime} \mathrm{f}_{\mathrm{m}-1-\mathrm{z}}^{\prime}-\left(A_{3} M+\frac{A_{1}}{\mathrm{Kp}}\right) \mathrm{f}_{\mathrm{m}-1}^{\prime}(\eta) \\
\mathcal{R}_{\mathrm{f}}^{\mathrm{m}} \theta_{\mathrm{m}}(\eta)=\frac{1}{A_{5}}\left[\frac{1}{P r} A_{4}+R d\right] \theta_{\mathrm{m}-1}^{\prime \prime}+\sum_{\mathrm{z}=0}^{\mathrm{m}-1} \mathrm{f}_{\mathrm{z}} \theta_{\mathrm{m}-1-\mathrm{z}}^{\prime}-2 \sum_{\mathrm{z}=0}^{\mathrm{m}-1} \theta_{\mathrm{z}} \mathrm{f}_{\mathrm{m}-1-\mathrm{z}}^{\prime}
\end{gathered}
$$

\section{Comparison of Results and Discussion}

The numerical and analytical solutions of shape effects of nanoparticles in Marangoni convection of graphene-water nanofluid under the influence of suction parameter are discussed here. The significant theme of the present section is to check the dimensionless physical quantities such as velocity and temperature of fluid for various values of pertinent parameters, namely suction parameter, permeability parameter, magmatic field, solid volume fraction, and radiation parameter.

Figure 1 presents the schematic model of the problem. Figure 2 reveals the effect of nanoparticles and also shows that the lamina-shaped nanoparticles have better performance in temperature distribution as compared to other shapes of nanoparticles in nanofluid. The reason is that the lamina-shaped nanoparticles have the lowest viscosity and thermal conductivity as compared to the other nanoparticle shapes, while an opposite performance is noted in sphere-shaped nanoparticles. The impacts of $\mathrm{M}$ on velocity and temperature of nanofluids are presented in Figure 3. It is clear from Figure 3 that the velocity of nanoparticles is inversely proportional but that the temperature is directly proportional to $\mathrm{M}$. This is due to Lorentz force which is induced by a magnetic field that opposes the motion of nanofluid and also Lorentz force heating in the energy equation, which assists as an increment in heat to the boundary layer. The lamina nanoparticles act as though they have a dominant role in temperature distribution with the impact of $\mathrm{M}$. The influences of $\mathrm{Kp}$ on velocity and temperature profiles are shown in Figure 4. This figure reveals that $\mathrm{Kp}$ has direct and inverse relations with velocity and temperature profiles, respectively. The reason is that with the enhancement of Kp, there is an increment in porous medium permeability which decreases the resistance in the nanofluid. As compared to other shapes of nanoparticles, lamina-shaped nanoparticles have a dominant role in temperature distribution under the impact of $\mathrm{Kp}$. The variation effects of $\varphi$ on velocity and temperature profiles are displayed in Figure 5. The velocity of nanofluid decreases, but the temperature 
profile intensifies with an increase in $\varphi$. The reason is that because of the nanoparticles, the thermal conductivity of the nanofluid increases which, in turn, boosts the thermal boundary layer thickness. The performance of lamina-shaped nanoparticles is dominant in temperature profiles with a variation in $\varphi$. Figure 6 describes the influences of A on velocity and temperature distribution and also shows that the velocity and temperature distribution of nanofluid decrease with intensifying of the suction parameter; physically, this happens because of the decreasing wall shear stress. The fact is that the heated nanofluid is pushed toward the wall where the buoyancy forces can increase the viscosity, so it decreases the wall shear stress. Under the influences of A, lamina-shaped nanoparticles have excellent performance in temperature profiles. Figure 7 portrays the potential of $\mathrm{Rd}$ on the temperature distribution and demonstrates that Rd has a direct relation with the temperature profile, physically, in the existence of thermal radiation significance increment in radiative heat, which assists in increasing the thermal stat of nanofluid, initiating its temperature to increase. It is also noted form Figure 7 that lamina-shaped nanoparticles show an effective role in temperature distribution. The performance of nanoparticles in the form of heat transfer is presented in Figures 8-13. From Figures 8-13, it is observed that the heat transfer rate of sphere shape nanoparticles in graphene-water is much better than for other nanoparticles. From Figures 8-13, it is noted that sphere $>$ platelet $>$ column $>$ lamina. The accuracy of the numerical results are checked by comparison with the analytical results which are obtained by the homotopy analysis method. In order to provide a comparison of the solution in a graphical form, Figures 14-17 were plotted. The validation of results for each nanoparticle shape is presented in Tables 3-6. Figure 18 displays the error decay for the 10th-order approximation.

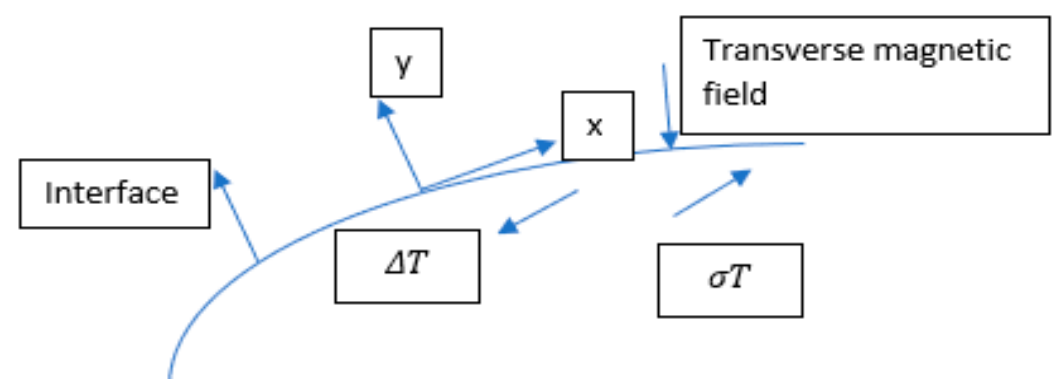

Figure 1. The schematic of the model.

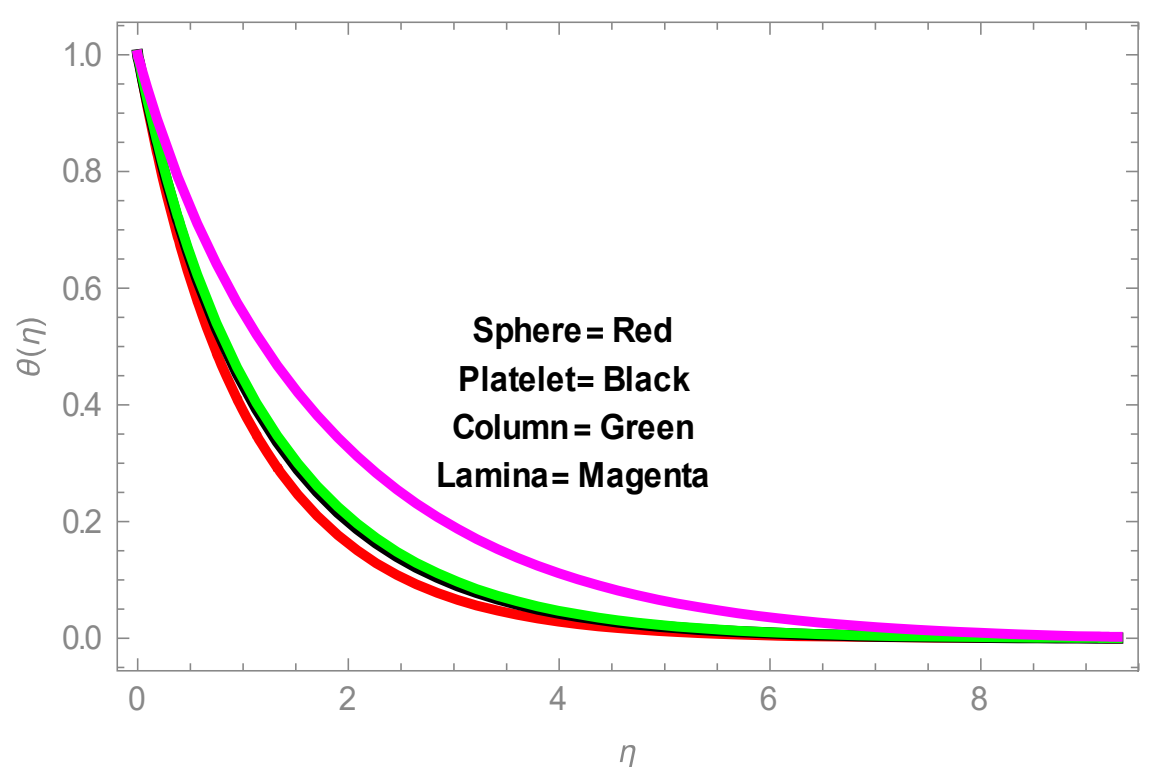

Figure 2. $\theta(\eta)$ for shapes of nanoparticles. 

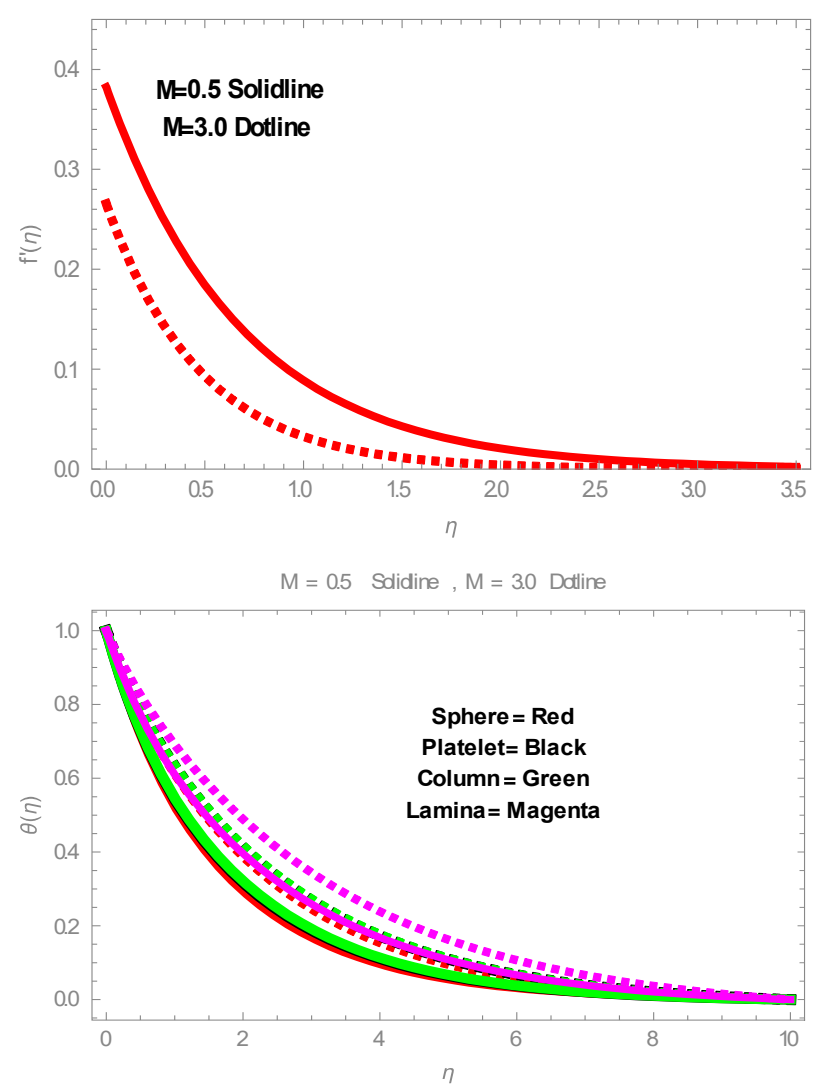

Figure 3. $f^{\prime}(\eta)$ and $\theta(\eta)$ for dissimilar values of $\mathrm{M}$.
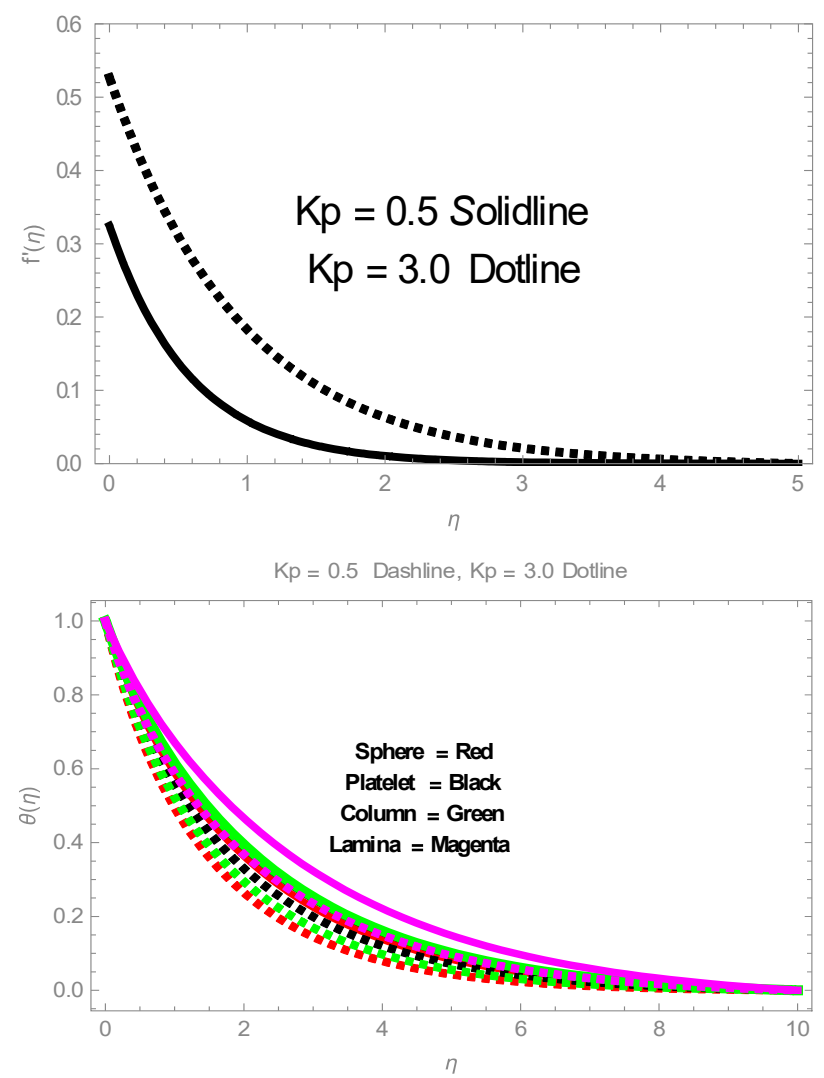

Figure 4. $f^{\prime}(\eta)$ and $\theta(\eta)$ for dissimilar values of Kp. 

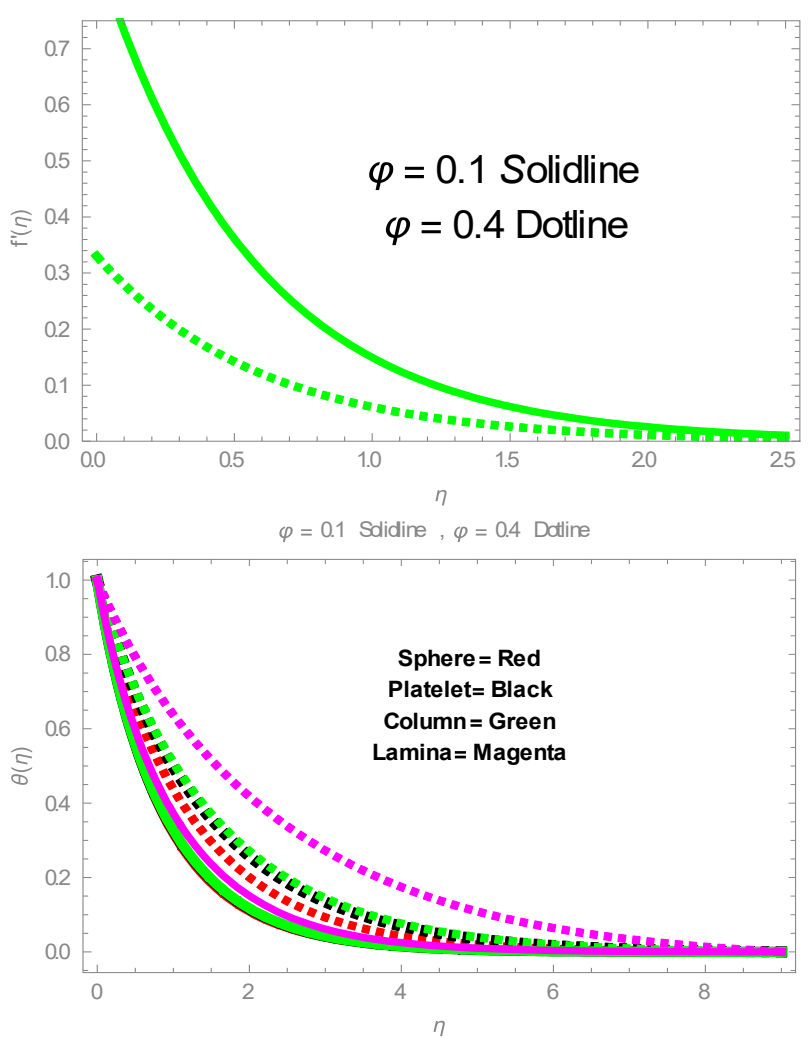

Figure 5. $f^{\prime}(\eta)$ and $\theta(\eta)$ for dissimilar values of $\phi$.
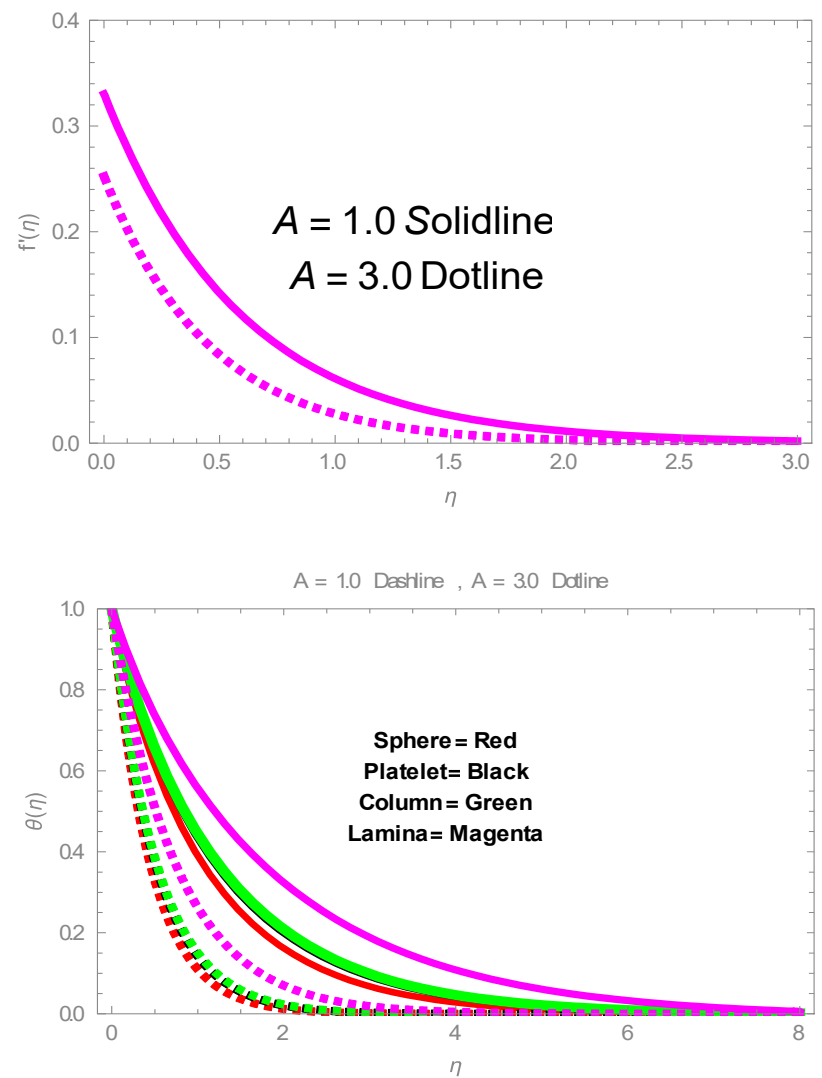

Figure 6. $f^{\prime}(\eta)$ and $\theta(\eta)$ for dissimilar values of A. 
$\mathrm{Rd}=1.0$ Solidline, $\mathrm{Rd}=3.0$ Dotline

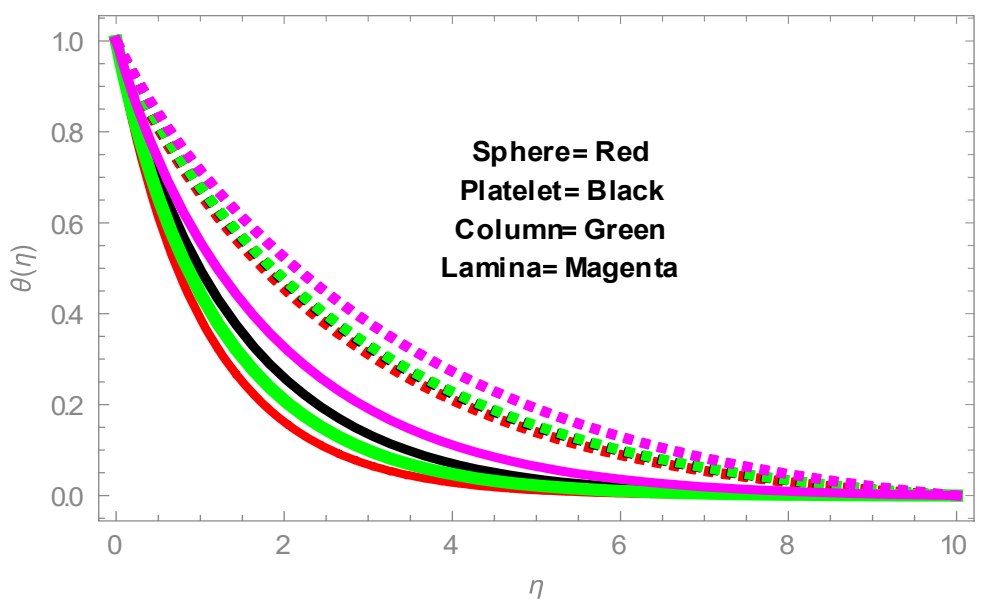

Figure 7. $\theta(\eta)$ for dissimilar values of $\mathrm{Rd}$.

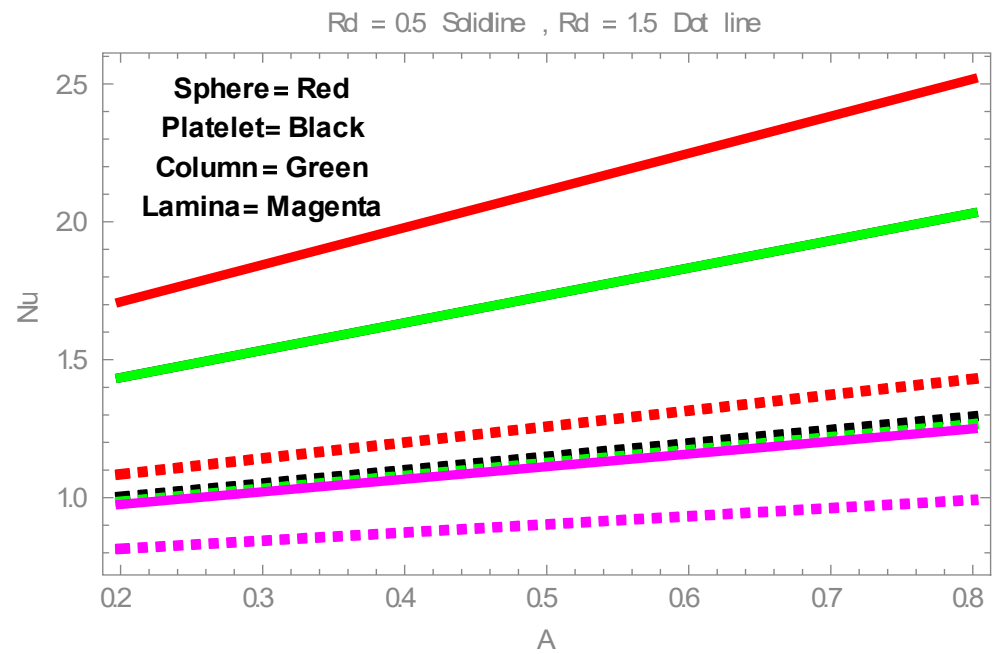

Figure 8. $\mathrm{Nu}$ for dissimilar values of $\mathrm{Rd}$.

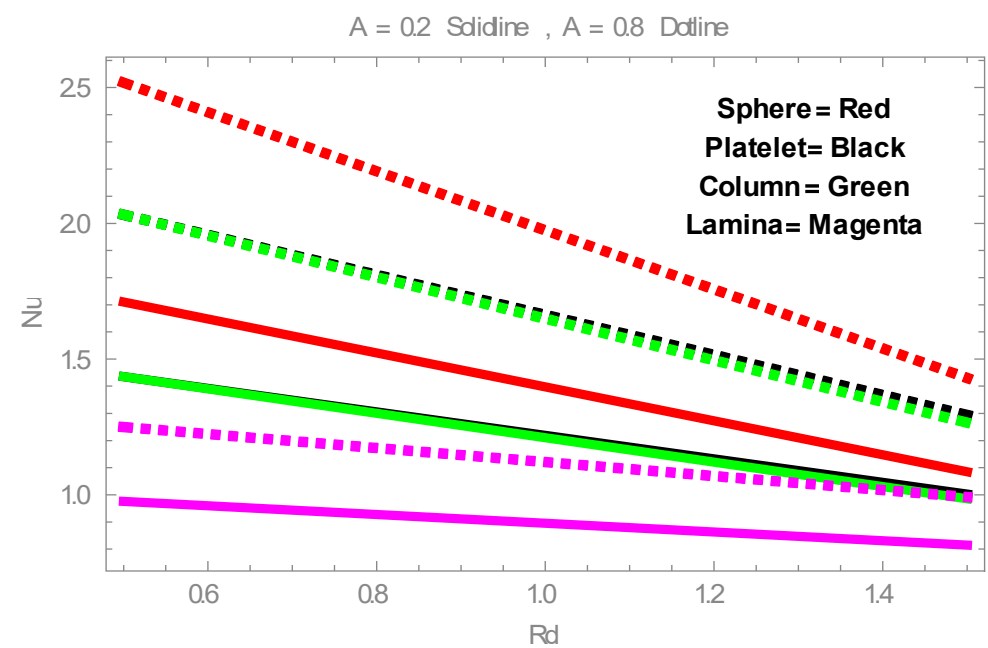

Figure 9. $\mathrm{Nu}$ for dissimilar values of $\mathrm{A}$. 
$K p=0.5$ Solidine,$K p=1.5$ Dotline

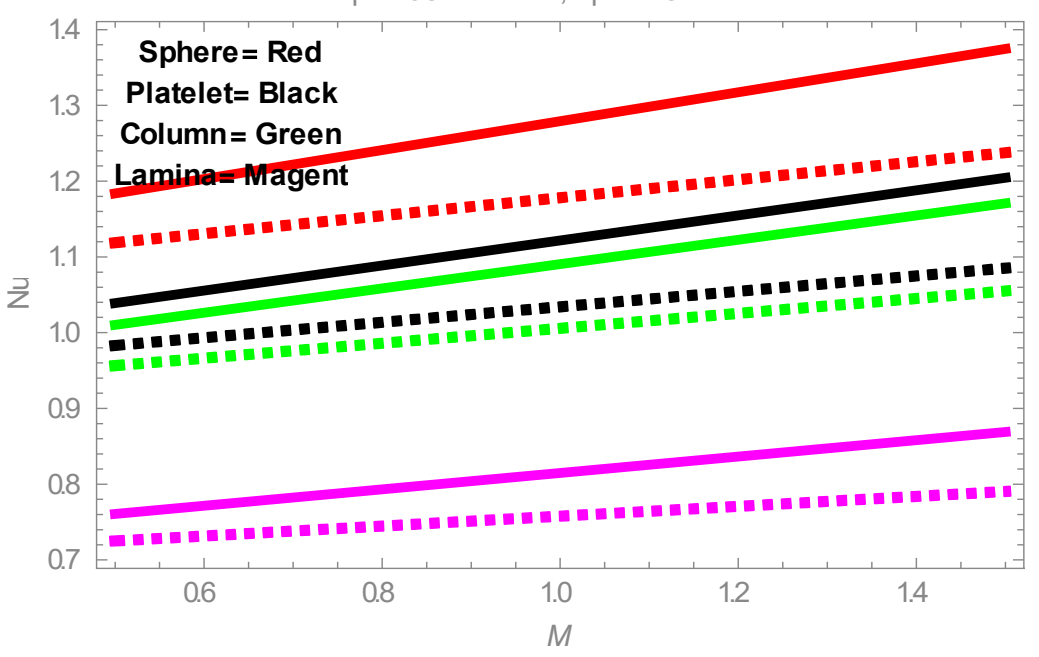

Figure 10. $\mathrm{Nu}$ for dissimilar values of $\mathrm{Kp}$.

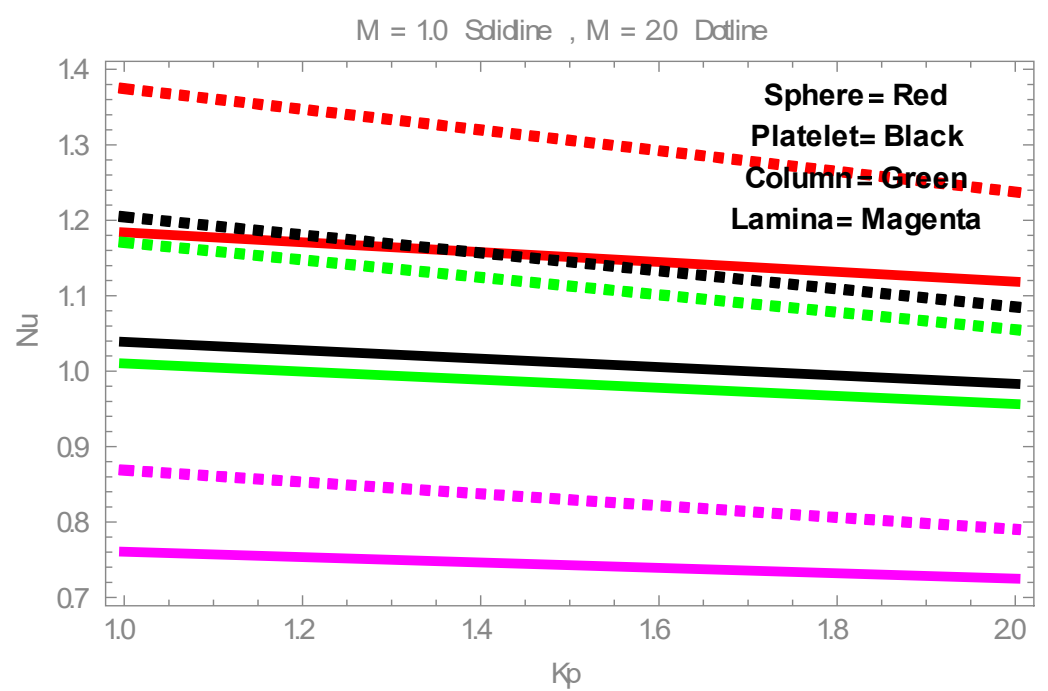

Figure 11. Nu for dissimilar values of M.

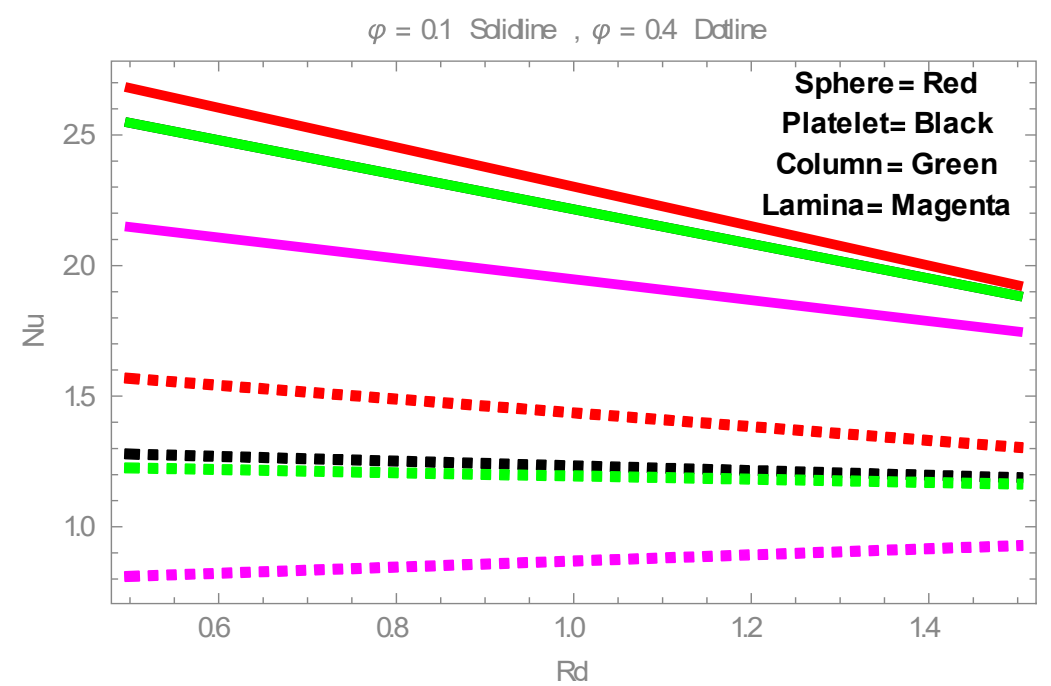

Figure 12. $\mathrm{Nu}$ for dissimilar values of $\phi$. 


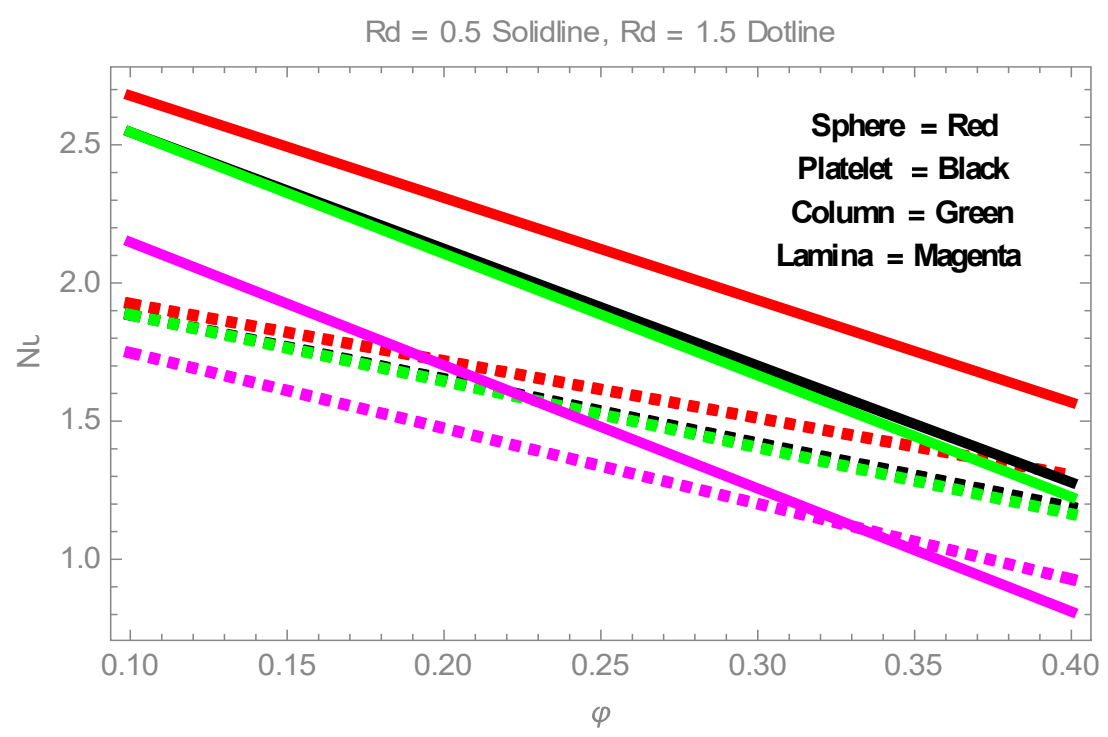

Figure 13. $\mathrm{Nu}$ for dissimilar values of $\mathrm{Rd}$

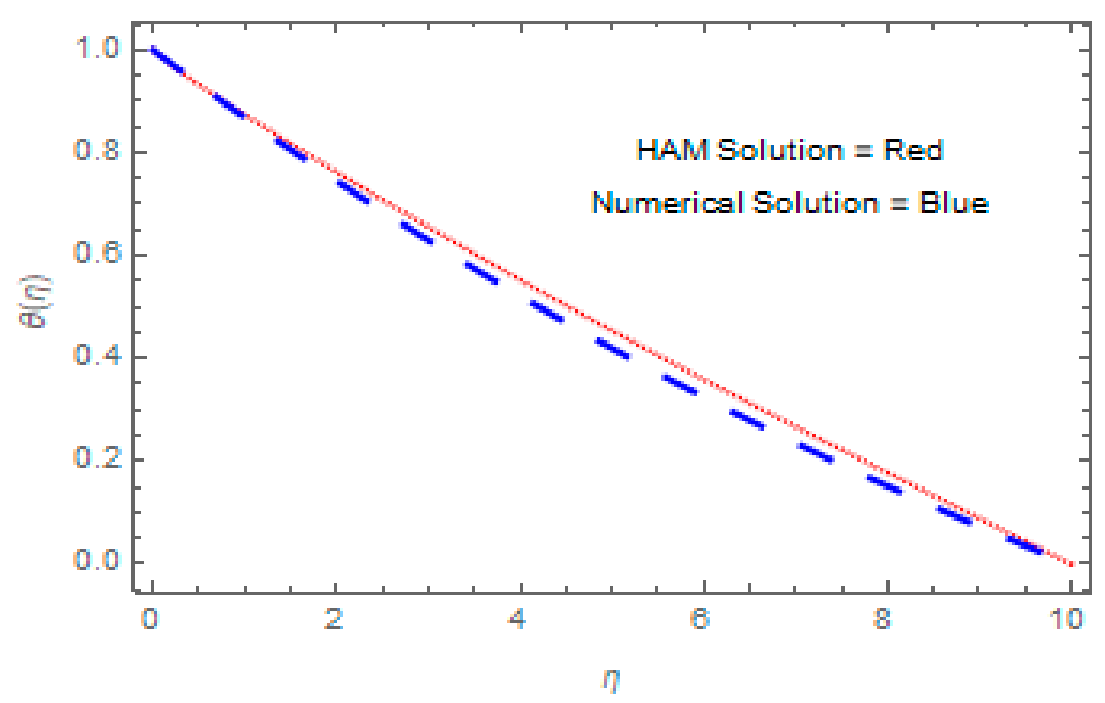

Figure 14. Comparison for sphere-shaped nanoparticles.

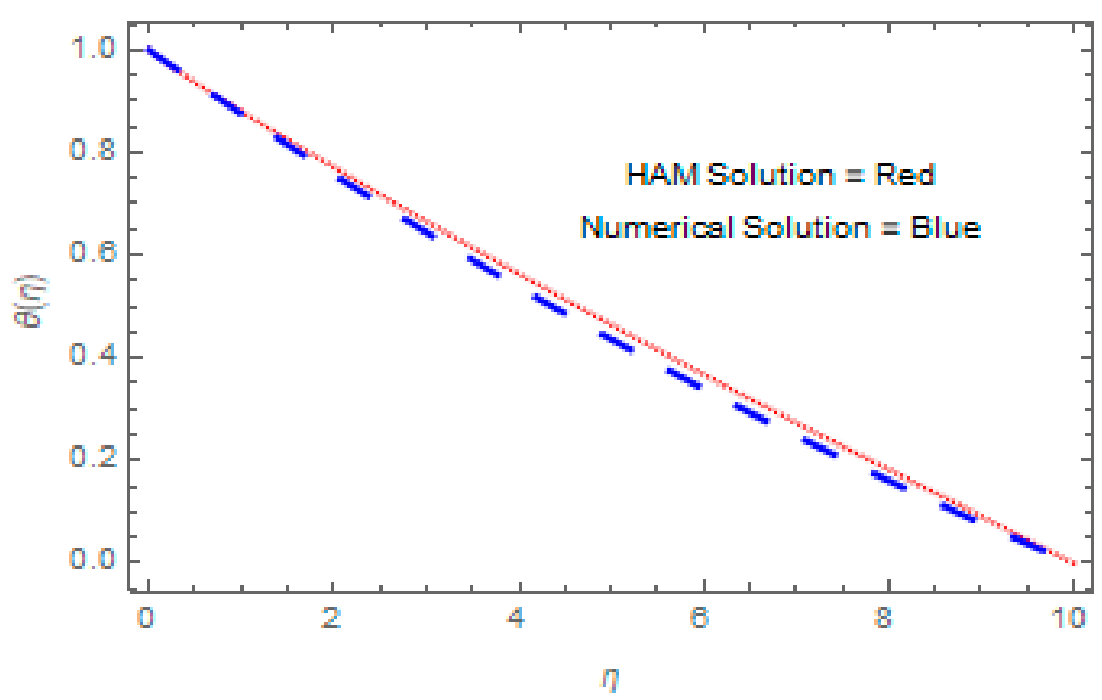

Figure 15. Comparison for platelet-shaped nanoparticles. 


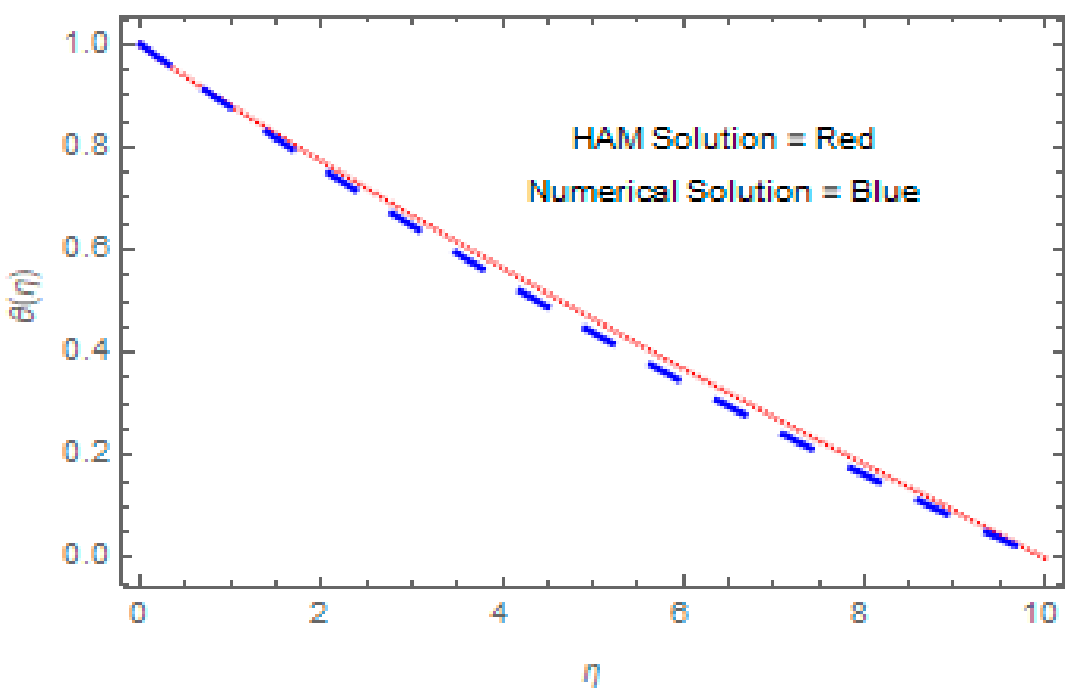

Figure 16. Comparison for column-shaped nanoparticles.

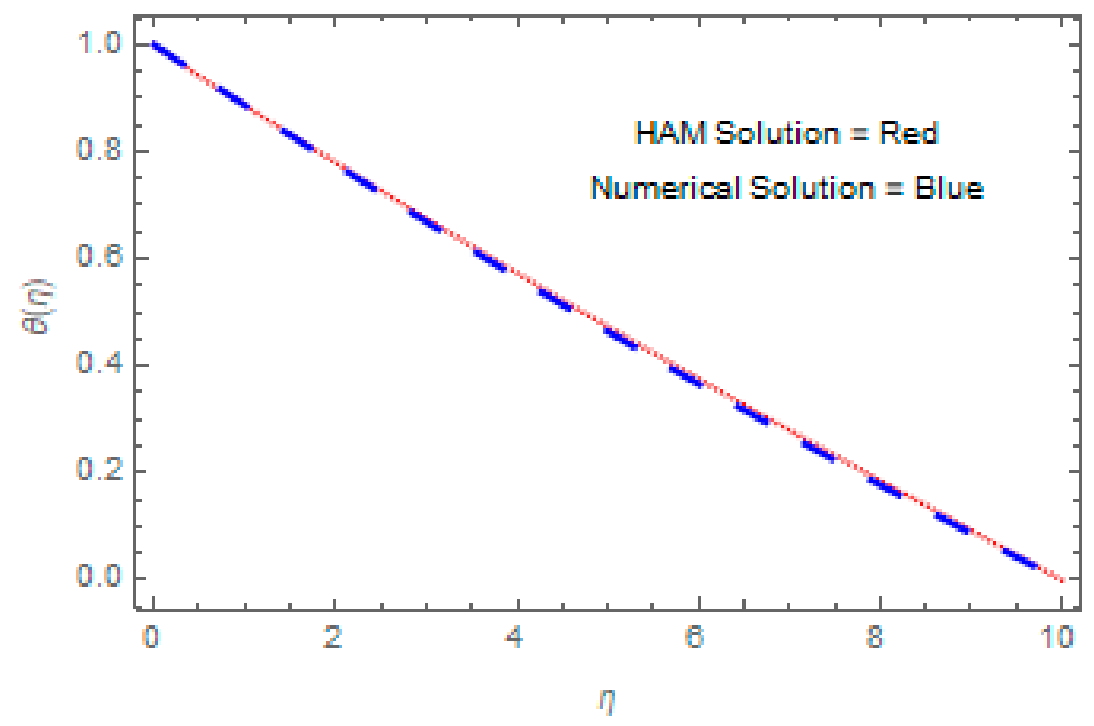

Figure 17. Comparison for lamina-shaped nanoparticles.

Table 3. Comparison results for sphere-shaped nanoparticles.

\begin{tabular}{cccc}
\hline$\eta$ & HAM Results & Numerical Results & Absolute Error \\
\hline 0 & 1.000000 & 1.000000 & 0.000000 \\
1 & 0.873762 & 0.868390 & 0.005372 \\
2 & 0.762481 & 0.745049 & 0.017432 \\
3 & 0.655491 & 0.629456 & 0.026035 \\
4 & 0.552687 & 0.521125 & 0.031562 \\
5 & 0.453964 & 0.419600 & 0.034365 \\
6 & 0.358922 & 0.324453 & 0.034469 \\
7 & 0.267190 & 0.235283 & 0.031907 \\
8 & 0.177983 & 0.511715 & 0.026268 \\
9 & 0.088937 & 0.073397 & 0.015540 \\
10 & 0.000045 & $-2.867620 \times 10^{-16}$ & 0.000048 \\
\hline
\end{tabular}


Table 4. Comparison results for platelet-shaped nanoparticles.

\begin{tabular}{cccc}
\hline $\boldsymbol{\eta}$ & HAM Results & Numerical Results & Absolute Error \\
\hline 0 & 1.000000 & 1.000000 & $2.22050 \times 10^{-16}$ \\
1 & 0.879780 & 0.875368 & 0.004413 \\
2 & 0.77126 & 0.756973 & 0.014289 \\
3 & 0.665913 & 0.644504 & 0.021409 \\
4 & 0.563643 & 0.537663 & 0.025970 \\
5 & 0.464400 & 0.436170 & 0.028230 \\
6 & 0.367944 & 0.339756 & 0.028187 \\
7 & 0.274100 & 0.248168 & 0.025932 \\
8 & 0.182831 & 0.161164 & 0.021668 \\
9 & 0.091886 & 0.078512 & 0.013374 \\
10 & 0.000045 & -0.000012 & 0.0000057 \\
\hline
\end{tabular}

Table 5. Comparison results for column-shaped nanoparticles.

\begin{tabular}{cccc}
\hline$\eta$ & HAM Results & Numerical Results & Absolute Error \\
\hline 0 & 1.00000 & 1.00000 & $1.110220 \times 10^{-16}$ \\
1 & 0.880803 & 0.876647 & 0.0004157 \\
2 & 0.772721 & 0.759171 & 0.013550 \\
3 & 0.667628 & 0.647293 & 0.020335 \\
4 & 0.565433 & 0.540745 & 0.024688 \\
5 & 0.466112 & 0.439275 & 0.026849 \\
6 & 0.369449 & 0.342639 & 0.026810 \\
7 & 0.275239 & 0.250608 & 0.024631 \\
8 & 0.183575 & 0.612962 & 0.020612 \\
9 & 0.92388 & 0.079491 & 0.012897 \\
10 & 0.000045 & -0.000015 & 0.0000060 \\
\hline
\end{tabular}

Table 6. Comparison results for lamina-shaped nanoparticles.

\begin{tabular}{cccc}
\hline $\boldsymbol{\eta}$ & HAM Results & Numerical Results & Absolute Error \\
\hline 0 & 1.00000 & 1.00000 & $1.110220 \times 10^{-16}$ \\
1 & 0.886622 & 0.886708 & 0.000085 \\
2 & 0.779944 & 0.776596 & 0.003348 \\
3 & 0.675410 & 0.669576 & 0.005834 \\
4 & 0.573134 & 0.565560 & 0.007574 \\
5 & 0.473222 & 0.464464 & 0.008757 \\
6 & 0.375596 & 0.366207 & 0.009272 \\
7 & 0.279981 & 0.270708 & 0.009272 \\
8 & 0.186039 & 0.177891 & 0.008148 \\
9 & 0.923294 & 0.087678 & 0.005616 \\
10 & 0.000045 & -0.000012 & 0.0000058 \\
\hline
\end{tabular}

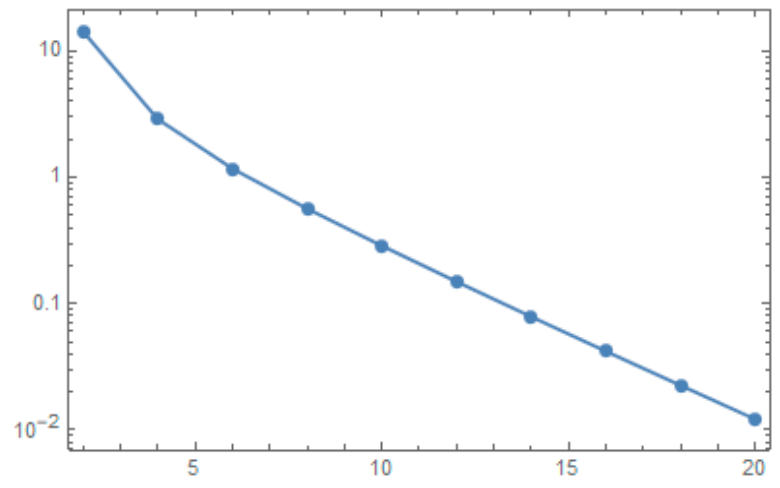

Figure 18. Error decay for the 10th-order approximation. 


\section{Concluding Remarks}

The shape effects of four different graphene nanoparticles (sphere, column, platelet, and lamina) on Marangoni boundary layer nanofluid flow and heat transfer with the effects of suction parameter were computed and discussed. On the basis of illustrative discussion, the following deductions are obtained:

- The accuracy of the numerical results has been verified with analytical results.

- The nanoparticles of lamina shape play a significant role in the disturbance of temperature profile.

- The nanoparticles of sphere shape play the smallest role in the disturbance of temperature profile.

- The nanoparticles of sphere shape play a remarkable role in the rate of heat transfer.

- The nanoparticles of lamina shape play the smallest role in the heat transfer rate.

Author Contributions: Conceptualization, U.R., H.L., A.I. and J.u.R.; methodology, U.R., D.B. and A.I.; software, U.R., M.A. and A.I.; validation, U.R., D.B., H.L., M.A. and J.u.R.; formal analysis, U.R., H.L., M.A., A.I. and J.u.R.; investigation, U.R., D.B., H.L., M.A., A.I. and J.u.R.; resources, D.B. and M.A.; writing-original draft preparation, U.R., M.A. and A.I.; writing-review and editing, U.R., D.B., H.L., M.A., A.I. and J.u.R.; visualization, D.B., M.A. and A.I.; supervision, D.B. and H.L.; funding acquisition, D.B. and M.A. All authors have read and agreed to the published version of the manuscript.

Funding: This research received no external funding.

Acknowledgments: The authors would like to thank the anonymous referees for their careful reading of this manuscript and also for their constructive suggestions which considerably improved the article. We also thank Muhammad Kashif Iqbal, GC University, Faisalabad, Pakistan for his assistance in proofreading the manuscript.

Conflicts of Interest: The authors declare no conflict of interest.

\section{Abbreviations}

The following abbreviations are used in this manuscript:

$\begin{array}{ll}\text { PDEs } & \text { Partial differential equations } \\ \text { ODEs } & \text { Ordinary differential equations } \\ \text { DEs } & \text { Differential equations } \\ \text { HAM } & \text { Homotopy analysis method }\end{array}$

\section{References}

1. Novoselov, K.; Geim, A.K.; Morozov, S.; Jiang, D.; Zhang, Y.; Dubonos, S.V.; Grigorieva, I.V.; Firsov, A.A. Electric Field Effect in Atomically Thin Carbon Films. Science 2004, 306, 666-669. [CrossRef]

2. Yu, X.; Wu, Q.; Zhang, H.; Zeng, G.; Li, W.; Qian, Y.; Li, Y.; Yang, G.; Chen, M. Investigation on synthesis, stability, and thermal conductivity properties of water-based $\mathrm{SnO}_{2} /$ reduced graphene oxide nanofluids. Materials 2017, 11, 38. [CrossRef]

3. Rehman, A.; Salleh, Z.; Gul, T.; Zaheer, Z. The Impact of Viscous Dissipation on the Thin Film Unsteady Flow of GO-EG/GO-W Nanofluids. Mathematics 2019, 7, 653. [CrossRef]

4. Aly, E.H. Dual exact solutions of graphene-water nanofluid flow over stretching/shrinking sheet with suction/injection and heat source/sink: Critical values and regions with stability. Powder Technol. 2019, 342, 528-544. [CrossRef]

5. Upadhya, S.M.; Raju, C.S.K.; Saleem, S.; Alderremy, A. Mahesha Modified Fourier heat flux on MHD flow over stretched cylinder filled with dust, Graphene and silver nanoparticles. Results Phys. 2018, 9, 1377-1385. [CrossRef]

6. Upadhya, S.M.; Mahesha; Raju, C.S.K. Unsteady Flow of Carreau Fluid in a Suspension of Dust and Graphene Nanoparticles With Cattaneo-Christov Heat Flux. J. Heat Transf. 2018, 140, 092401. [CrossRef]

7. Khan, N.S.; Zuhra, S. Boundary layer flow and heat transfer in a thin-film second-grade nanoliquid embedded with graphene nanoparticles. Adv. Mech. Eng. 2019, 11,1-11. [CrossRef]

8. Shit, G.C.; Mukherjee, S. MHD graphene-polydimethylsiloxane Maxwell nanofluid flow in a squeezing channel with thermal radiation effects. Appl. Math. Mech. 2019, 40, 1269-1284. [CrossRef] 
9. Arif, M.; Ali, F.; Sheikh, N.A.; Khan, I. Enhanced heat transfer in working fluids using nanoparticles with ramped wall temperature: Applications in engine oil. Adv. Mech. Eng. 2019, 11, 1-11. [CrossRef]

10. Periasamy, S.M.; Baskar, R. Assessment of the Influence of Graphene Nanoparticles on Thermal Conductivity of Graphene/Water Nanofluids Using Factorial Design of Experiments. Period. Polytech. Chem. Eng. 2018, 62, 317-322. [CrossRef]

11. Azimi, M.; Riazi, R. Heat transfer analysis of GO-water nanofluid flow between two parallel disks. Propuls. Power Res. 2015, 4, 23-30. [CrossRef]

12. Moosavi, M.; Momeni, M.; Tavangar, T.; Mohammadyari, R.; Rahimi-Esbo, M. Variational iteration method for flow of non-Newtonian fluid on a moving belt and in a collector. Alex. Eng. J. 2016, 55, 1775-1783. [CrossRef]

13. Maghsoudi, P.; Shahriari, G.; Rasam, H.; Sadeghi, S. Flow and natural convection heat transfer characteristics of non-Newtonian nanofluid flow bounded by two infinite vertical flat plates in presence of magnetic field and thermal radiation using Galerkin method. J. Cent. South Univ. 2019, 26, 1294-1305. [CrossRef]

14. Aliy, G.; Kishan, N. Optimal Homotopy Asymptotic Solution for Cross-Diffusion Effects on Slip Flow and Heat Transfer of Electrical MHD Non-Newtonian Fluid Over a Slendering Stretching Sheet. Int. J. Appl. Comput. Math. 2019, 5, 80. [CrossRef]

15. Freidoonimehr, N.; Rostami, B.; Rashidi, M. Predictor homotopy analysis method for nanofluid flow through expanding or contracting gaps with permeable walls. Int. J. Biomath. 2015, 8, 1550050. [CrossRef]

16. Usman, M.; Hamid, M.; Khan, U.; Din, S.T.M.; Iqbal, M.A.; Wang, W. Differential transform method for unsteady nanofluid flow and heat transfer. Alex. Eng. J. 2018, 57, 1867-1875. [CrossRef]

17. Eldabe, N.; Abou-Zeid, M. Homotopy perturbation method for MHD pulsatile non-Newtonian nanofluid flow with heat transfer through a non-Darcy porous medium. J. Egypt. Math. Soc. 2017, 25, 375-381. [CrossRef]

18. Bakodah, H.O.; Ebaid, A. The Adomian decomposition method for the slip flow and heat transfer of nanofluids over a stretching/shrinking sheet. Rom. Rep. Phys. 2018, 70, 115.

19. Al-Mudhaf, A.; Chamkha, A.J. Similarity solutions for MHD thermosolutal Marangoni convection over a flat surface in the presence of heat generation or absorption effects. Heat Mass Transf. 2005, 42, 112-121. [CrossRef]

20. Zhang, Y.; Zheng, L.; Liu, J. Approximate Analytical Solutions for Marangoni Mixed Convection Boundary Layer. In Proceedings of the 4th International Heat Transfer Conference, Washington, DC, USA, 8-13 August 2010; pp. 413-417. [CrossRef]

21. Christopher, D.; Wang, B.-X. Marangoni Convection Around a Bubble In Microgravity. Heat Transf. 1998, 3, 489-494. [CrossRef]

22. Straub, J. The role of surface tension for two-phase heat and mass transfer in the absence of gravity. Exp. Therm. Fluid Sci. 1994, 9, 253-273. [CrossRef]

23. Pop, I.; Postelnicu, A.; Grosan, T. Thermosolutal Marangoni Forced Convection Boundary Layers. Meccanica 2001, 36, 555-571. [CrossRef]

24. Golia, C.; Viviani, A. Non isobaric boundary layers related to Marangoni flows. Meccanica 1986, 21, $200-204$. [CrossRef]

25. Christopher, D.M.; Wang, B. Prandtl number effects for Marangoni convection over a flat surface. Int. J. Therm. Sci. 2001, 40, 564-570. [CrossRef]

26. Chamkha, A.J.; Pop, I.; Takhar, H. Marangoni Mixed Convection Boundary Layer Flow. Meccanica 2006, 41, 219-232. [CrossRef]

27. Magyari, E.; Chamkha, A. Exact analytical results for the thermosolutal MHD Marangoni boundary layers. Int. J. Therm. Sci. 2008, 47, 848-857. [CrossRef]

28. Arafune, K.; Hirata, A. Thermal and solutal Marangoni convection in In-Ga-Sb system. J. Cryst. Growth 1999, 197, 811-817. [CrossRef]

29. Lin, Y.; Li, B.; Zheng, L.; Chen, G. Particle shape and radiation effects on Marangoni boundary layer flow and heat transfer of copper-water nanofluid driven by an exponential temperature. Powder Technol. 2016, 301, 379-386. [CrossRef]

30. Aly, E.; Ebaid, A. Exact analysis for the effect of heat transfer on MHD and radiation Marangoni boundary layer nanofluid flow past a surface embedded in a porous medium. J. Mol. Liq. 2016, 215, 625-639. [CrossRef] 
31. Hayat, T.; Khan, M.I.; Farooq, M.; Alsaedi, A.; Yasmeen, T. Impact of Marangoni convection in the flow of carbon-water nanofluid with thermal radiation. Int. J. Heat Mass Transf. 2017, 106, 810-815. [CrossRef]

32. Sheikholeslami, M.; Chamkha, A.J. Influence of Lorentz forces on nanofluid forced convection considering Marangoni convection. J. Mol. Liq. 2017, 225, 750-757. [CrossRef]

33. Bognár, G.; Hriczó, K. Series Solutions for Marangoni Convection on a Vertical Surface. Math. Probl. Eng. 2012, 2012, 1-18. [CrossRef]

34. Yan, Z.; Liancun, Z.; Xiaojing, W.; Guhua, S. Analysis of Marangoni convection of non-Newtonian power law fluids with linear temperature distribution. Therm. Sci. 2011, 15, 45-52. [CrossRef]

35. Lin, Y.; Zheng, L. Marangoni boundary layer flow and heat transfer of copper-water nanofluid over a porous medium disk. AIP Adv. 2015, 5, 107225. [CrossRef]

36. Kandasamy, R.; Adnan, N.A.B.; Mohammad, R. Nanoparticle shape effects on squeezed MHD flow of water based $\mathrm{Cu}, \mathrm{Al}_{2} \mathrm{O}_{3}$ and SWCNTs over a porous sensor surface. Alex. Eng. J. 2018, 57, 1433-1445. [CrossRef]

37. Rashid, U.; Ibrahim, A. Impacts of Nanoparticle Shape on $\mathrm{Al}_{2} \mathrm{O}_{3}$-Water Nanofluid Flow and Heat Transfer over a Non-Linear Radically Stretching Sheet. Adv. Nanopart. 2020, 9, 23-39. [CrossRef]

38. Aman, S.; Khan, I.; Ismail, Z.; Salleh, M.Z. Impacts of gold nanoparticles on MHD mixed convection Poiseuille flow of nanofluid passing through a porous medium in the presence of thermal radiation, thermal diffusion and chemical reaction. Neural Comput. Appl. 2016, 30, 789-797. [CrossRef]

(C) 2020 by the authors. Licensee MDPI, Basel, Switzerland. This article is an open access article distributed under the terms and conditions of the Creative Commons Attribution (CC BY) license (http://creativecommons.org/licenses/by/4.0/). 